\title{
FGF10 missense mutations in aplasia of lacrimal and salivary glands (ALSG)
}

\author{
Miriam Entesarian ${ }^{1}$, Johanna Dahlqvist ${ }^{1}$, Vandana Shashi ${ }^{2}$, Christy S Stanley ${ }^{2}$, \\ Babak Falahat ${ }^{3}$, William Reardon ${ }^{4}$ and Niklas Dahl ${ }^{*, 1}$
}

\begin{abstract}
${ }^{1}$ The Rudbeck Laboratory, Department of Genetics and Pathology, Uppsala University, Uppsala SE-751 85, Sweden; ${ }^{2}$ Department of Pediatrics, Medical Genetics, Wake Forest University, Winston-Salem, NC, USA; ${ }^{3}$ Department of Maxillofacial Radiology, Institute for Postgraduate Dental Education, Jönköping, Sweden; ${ }^{4}$ National Center for Medical Genetics, Our Lady's Children's Hospital, Dublin, Ireland
\end{abstract}

Aplasia of lacrimal and salivary glands (ALSG) is an autosomal dominant congenital anomaly characterized by aplasia, atresia or hypoplasia of the lacrimal and salivary systems. Affected individuals present with irritable eyes and dryness of the mouth with variable expressivity. Mutations in FGF10 were recently described in ALSG and in lacrimo-auriculo-dento-digital (LADD) syndrome which are overlapping clinical entities. We present here two families with ALSG associated with missense mutations (R80S and G138E, respectively) affecting highly conserved residues in FGF10. The clinical features of these patients further broaden the knowledge of FGF10-related phenotypes.

European Journal of Human Genetics (2007) 15, 379-382. doi:10.1038/sj.ejhg.5201762; published online 10 January 2007

Keywords: ALSG; LADD syndrome; FGF10; missense mutations

\section{Introduction}

Aplasia of lacrimal and major salivary glands (ALSG; OMIM 180920) is a rare autosomal dominant disorder with variable expressivity. Aplasia of the major salivary and lacrimal glands may be associated with atresia of the nasolacrimal duct and absence of lacrimal puncta. The clinical manifestations vary but involvement of the lacrimal glands results in irritable eyes, recurrent eye infections and epiphora (constant tearing) if the nasolacrimal ducts or lacrimal puncta are missing. ${ }^{1}$ Aplasia or hypoplasia of the major salivary glands causes xerostomia (dryness of the mouth), which increases the risk of dental erosion and dental caries. ${ }^{2}$ Other complications include periodontal disease, oral soft tissue inflammation and disorders of smell, chewing and swallowing. ${ }^{3}$ We previously

*Correspondence: Professor N Dahl, Department of Genetics and Pathology, Uppsala University Hospital, Unit of Clinical Genetics, Rudbeck Laboratory, Dag Hammarsköldsväg 20, Uppsala SE-751 85, Sweden. Tel: + 4618611 2799; Fax: + 4618554 025;

E-mail: niklas.dahl@genpat.uu.se

Received 21 August 2006; revised 2 November 2006; accepted 8 November 2006; published online 10 January 2007 identified loss of function mutations in the fibroblast growth factor 10 (FGF10) gene associated with ALSG. ${ }^{1}$ More recent studies have shown ALSG to be allelic to lacrimo-auriculo-dento-digital (LADD; OMIM 149730) syndrome, which is a similar clinical entity. ${ }^{4,5}$ LADD syndrome is an autosomal dominant disorder characterized by aplasia or hypoplasia of the lacrimal and salivary systems as well as abnormalities of the face, ears, eyes, mouth, teeth, digits and genitourinary system. Moreover, LADD syndrome is genetically heterogeneous and may also be caused by mutations in the fibroblast growth factor receptors 2 and 3 (FGFR2, FGFR3). ${ }^{4}$ We have examined two non-related individuals with ALSG and we report for the first time that FGF10 missense mutations are associated with this clinical entity.

\section{Patients and methods \\ Patients}

The proband from family 1 is a three-year-old boy and the only child of non-related parents of Caucasian origin (Figure 1). Prenatal ultrasounds were normal. The boy was born at gestational week 37 after a normal pregnancy and 


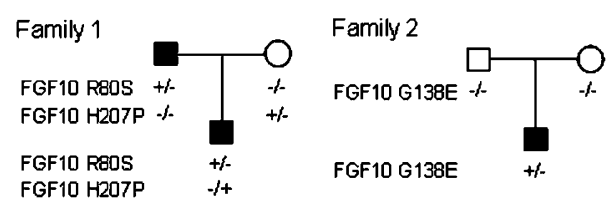

Figure 1 Pedigrees of the two families with ALSG and predicted a.a. substitutions in FGF10. Filled symbols denote affected individuals. + , presence; - , absence.

delivery. The birth weight was $2637 \mathrm{~g}$ (-2 SD), length $47 \mathrm{~cm}$ ( $-1 \mathrm{SD})$ and head circumference $35 \mathrm{~cm}$ (0SD). Apgar scores were 9 after 1 and $5 \mathrm{~min}$, respectively. The parents noticed that he never produced tears when crying and that he did not drool as a baby. When eating he has to take frequent sips of water to swallow the food. Fluorescent staining of the eyes displayed mild abrasions of the cornea. Lacrimal fluid production tests (Schirmer test) established a reduced tear production. The lacrimal puncta were not visible. Oral examination of the patient confirmed dry mucosa. He has had four dental cavities at age 3 years. Sjögren syndrome was excluded by serology testing. The father has similar features, with absent tear production, dry mouth and difficulties in swallowing. The father has had several dental cavities followed by extensive dental care. He does not produce tears. Magnetic resonance imaging (MRI) of the lacrimal and salivary glands on the father revealed bilaterally absent lacrimal glands and hypoplastic submandibular glands. The parotid glands were present but very fatty in appearance. Both father and son have normal hearing and clinical examination of the proband including the digits, ears, primary teeth and external genitalia revealed no abnormalities. The parents elected for the child not to have an MRI. The mother is healthy and has no signs of either ALSG or LADD syndrome.

The proband from family 2 is a 4 -year-old boy and the first child of healthy, non-related parents of Caucasian origin (Figure 1). The pregnancy and delivery at gestational week 40 were normal. His birth weight was $3135 \mathrm{~g}$ (-1SD) and length $53 \mathrm{~cm}$ (0SD). Apgar score was 7. The patient had coronal hypospadias but no other genitourinary abnormalities. With age, the parents noticed that the boy did not produce tears when crying. Clinical examination at 4 years of age revealed absent inferior lacrimal puncta. Schirmer's test indicated lacrimal production within the normal range. Oral examination showed signs of hyposalivation including dental caries, irritated oral mucosa, fissured tongue and dry lips. Autoantibody analysis for Sjögren syndrome was normal. MRI revealed aplasia of lacrimal, parotid and submandibular glands. Sublingual glands were present and of normal size. The boy has no detectable anomalies involving the digits, ears or primary teeth. He has no hearing loss.

Informed consent was obtained from parents of both families. This study is approved by the regional ethical committee of Uppsala.

\section{PCR and sequence analysis}

Blood samples were obtained from the two probands and their parents for DNA extraction and genetic analysis. The three exons of FGF10 as well as the exon-intron boundaries were amplified and bidirectionally sequenced, using Big Dye Terminator v 3.1 Cycle Sequencing chemistry (Applied Biosystems) and the ABI 3700 DNA Analyzer (Applied Biosystems), according to the manufacturer's recommendations. Base calling was performed with the DNA Sequencing Analysis software, version 3.7 (Applied Biosystems) and the DNA sequences were aligned and analyzed with the Sequencher software, version 4.1 (Gene Codes Corporation). For paternity testing we analyzed 16 polymorphic microsatellite markers (Powerplex16, Promega). The ConSeq web server was used to identify biologically important amino-acid residues in FGF10. ${ }^{6}$ Control DNA was obtained from healthy Caucasian blood donors.

\section{Results}

FGF10 gene analysis of the proband from family 1 revealed two missense mutations. One is a heterozygous $240 \mathrm{~A}>\mathrm{C}$ nucleotide transversion in exon 1, predicted to result in a chemically non-conservative (basic to neutral) amino-acid substitution from arginine to serine at position 80 (Figure 2). This residue is highly conserved. The nucleotide transversion was also present in DNA from the affected father but it was not observed in 308 control chromosomes. An additional heterozygous $620 \mathrm{~A}>\mathrm{C}$ nucleotide transversion was identified in DNA of the proband that was inherited from the mother. This nucleotide transversion is located in exon 3 of FGF1O and is predicted to result in a non-conservative amino-acid change from histidine to proline (basic to neutral) at position 207 (Figure 2). This residue is not evolutionary conserved. The $620 \mathrm{~A}>\mathrm{C}$ substitution was not identified in 330 control chromosomes and it could not be confirmed as a polymorphism.

Sequence analysis of FGF10 in the proband and healthy parents of family 2 disclosed a heterozygous nucleotide transition, $413 \mathrm{G}>\mathrm{A}$, in exon 2 in the proband only. Paternity was confirmed by microsatellite analysis, which indicated that the mutation had occurred de novo. The $413 \mathrm{G}>\mathrm{A}$ transition results in a non-conservative substitution from glycine to glutamic acid (neutral to acidic) at position 138 of FGF10 (Figure 2). The transition affects a highly conserved residue and was absent in 296 control chromosomes.

\section{Discussion}

We and others have recently shown that both ALSG and LADD syndrome are caused by dominant acting FGF10 gene mutations. ${ }^{1,4,5}$ To date, ALSG has exclusively been associated with loss of function mutations in FGF10. This suggests that haploinsufficiency during a critical stage of 


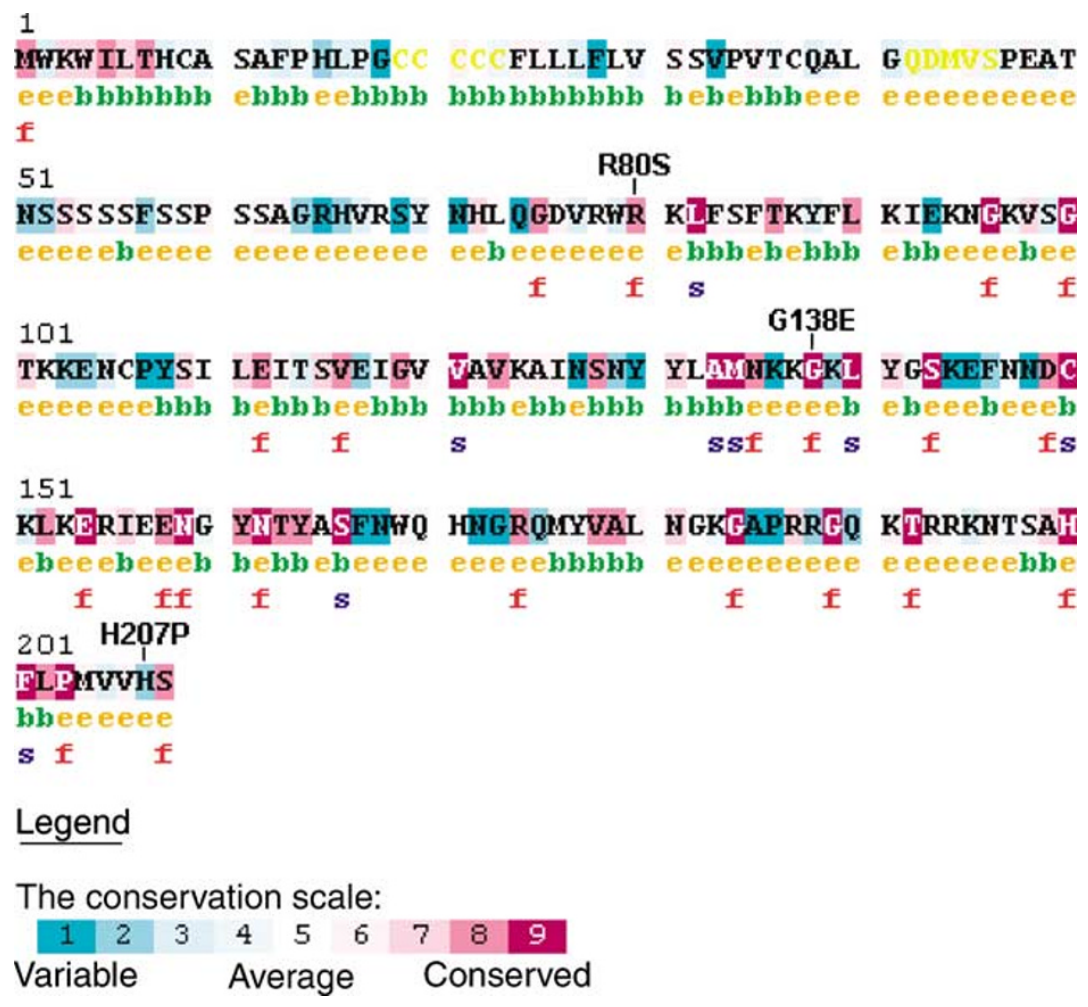

Figure 2 Locations of ALSG associated mutations in FGF10. ConSeq predictions visualize the amino-acid conservation grade in colour-code. The predicted status of each residue, buried (b) or exposed (e), is marked below the amino-acid sequence. Slowly evolving and exposed residues are predicted to be functional $(\mathrm{f})$, whereas slowly evolving and buried residues are predicted to be structurally important (s). The residues 80 and 138 are highly conserved in contrast to residue 207.

development causes ALSG. This is also supported by studies in mice which demonstrate that two copies of Fgf1O are required for the normal development of lacrimal and salivary glands. ${ }^{1}$ Two missense mutations have previously been reported in FGF10 and in both cases associated with LADD syndrome. ${ }^{4,5}$ In this study, we report for the first time a FGF10 missense mutation associated with isolated ALSG.

In family 1, a father and his son are affected by ALSG and they both carry an R80S substitution in FGF10. The arginine at position 80 in FGF10 is known to interact with the D3 region of FGFR2b. ${ }^{7}$ The amino-acid substitution may alter or abolish the interaction between FGF10 and FGFR2b, which would result in aberrant or insufficient signal transduction. Interestingly, the proband from family 1 also carries an H207P substitution inherited from the healthy mother. This residue is not predicted to be evolutionary conserved (Figure 2). Neither of the two mutations was found upon screening over 300 control chromosomes. The fact that both father and son have very similar clinical signs and the absence of any ALSG symptoms in the mother suggests the H207P substitution to be a rare polymorphism.

In family 2, we identified a sporadic case with a glycine to glutamic acid substitution at position 138 in FGF10, which is a highly conserved amino-acid residue of predicted functional importance (Figure 2). The parents do not present with any symptoms of ALSG and DNA analysis confirmed G138E as a de novo mutation. The proband of family 2 also has coronal hypospadias. Urogenital malformations, including hypospadias, have previously been described in some patients with LADD syndrome. ${ }^{8}$ Consequently, the phenotype of this proband bridges the characteristics of ALSG and LADD syndrome. This further supports the significant clinical overlap between ALSG and LADD syndrome in addition to their common features of aplasia, atresia or hypoplasia of the lacrimal and salivary systems.

Our findings indicate that ALSG may be associated with missense mutations affecting conserved or functionally important residues in FGF10. It has been hypothesized that ALSG results from haploinsufficiency of FGF10 whereas LADD syndrome is caused by a more complex mechanism involving residual activity derived from the mutant allele. However, this is contradicted by the finding by Milunsky et al. ${ }^{5}$ of a nonsense mutation in FGF10 in a mother with ALSG and her daughter with LADD syndrome. This variability in clinical expression is more likely to be explained by modifier gene loci such as FGFR2 and FGFR3 or, stochastic events. 
Identification of additional FGF10 mutations associated with variable expression of ALSG and LADD syndrome may result in increased diagnostic accuracy with implications for genetic counselling. The identification of additional ALSG as well as LADD syndrome patients with FGF10 gene mutations will further clarify genotype-phenotype relationships and how they affect FGF10 signalling.

\section{Acknowledgements}

We thank the patients and their families for their contribution to this study. This work was supported by grants from the Swedish Research Council, the Thuréus Foundation, the Children's Cancer Foundation of Sweden, Uppsala University and the Torsten and Ragnar Söderbergs Fund.

\section{References}

1 Entesarian $\mathrm{M}$, Matsson $\mathrm{H}$, Klar $\mathrm{J}$ et al: Mutations in the gene encoding fibroblast growth factor 10 are associated with aplasia of lacrimal and salivary glands. Nat Genet 2005; 37: 125-127.
2 Young W, Khan F, Brandt R, Savage N, Razek AA, Huang Q: Syndromes with salivary dysfunction predispose to tooth wear: case reports of congenital dysfunction of major salivary glands, Prader-Willi, congenital rubella, and Sjogren's syndromes. Oral Surg Oral Med Oral Pathol Oral Radiol Endod 2001; 92: 38-48.

3 Ferreira AP, Gomez RS, Castro WH, Calixto NS, Silva RA, Aguiar MJ: Congenital absence of lacrimal puncta and salivary glands: report of a Brazilian family and review. Am J Med Genet 2000; 94: $32-34$.

4 Rohmann E, Brunner HG, Kayserili $\mathrm{H}$ et al: Mutations in different components of FGF signaling in LADD syndrome. Nat Genet 2006; 38: $414-417$.

5 Milunsky JM, Zhao G, Maher TA, Colby R, Everman DB: LADD syndrome is caused by FGF10 mutations. Clin Genet 2006; 69: 349-354.

6 Berezin C, Glaser F, Rosenberg J et al: ConSeq: the identification of functionally and structurally important residues in protein sequences. Bioinformatics 2004; 20: 1322-1324.

7 Yeh BK, Igarashi M, Eliseenkova AV et al: Structural basis by which alternative splicing confers specificity in fibroblast growth factor receptors. Proc Natl Acad Sci USA 2003; 100: 2266-2271.

8 Bamforth JS, Kaurah P: Lacrimo-auriculo-dento-digital syndrome: evidence for lower limb involvement and severe congenital renal anomalies. Am J Med Genet 1992; 43: 932-937. 